\title{
The role of $\mathrm{Cra}$ in regulating acetate excretion and osmotic tolerance in E. coli $\mathrm{K}-12$ and E. coli B at high density growth
}

\author{
Young-Jin Son ${ }^{1 \dagger}$, Je-Nie Phue ${ }^{1 \dagger}$, Loc B Trinh', Sang Jun Lee ${ }^{2}$ and Joseph Shiloach ${ }^{1 *}$
}

\begin{abstract}
Background: E. coli B (BL21), unlike E.coli K-12 (JM109) is insensitive to glucose concentration and, therefore, grows faster and produces less acetate than E. coli K-12, especially when growing to high cell densities at high glucose concentration. By performing genomic analysis, it was demonstrated that the cause of this difference in sensitivity to the glucose concentration is the result of the differences in the central carbon metabolism activity. We hypothesized that the global transcription regulator Cra (FruR) is constitutively expressed in E. coli B and may be responsible for the different behaviour of the two strains. To investigate this possibility and better understand the function of Cra in the two strains, cra - negative E. coli B (BL21) and E. coli K-12 (JM109) were prepared and their growth behaviour and gene expression at high glucose were evaluated using microarray and real-time PCR.

Results: The deletion of the cra gene in E. coli B (BL21) minimally affected the growth and maximal acetate accumulation, while the deletion of the same gene in E.coli K-12 (JM109) caused the cells to stop growing as soon as acetate concentration reached $6.6 \mathrm{~g} / \mathrm{L}$ and the media conductivity reached $21 \mathrm{mS} / \mathrm{cm}$. ppsA (gluconeogenesis gene), aceBA (the glyoxylate shunt genes) and poxB (the acetate producing gene) were down-regulated in both strains, while acs (acetate uptake gene) was down-regulated only in E.coli B (BL21). These transcriptional differences had little effect on acetate and pyruvate production. Additionally, it was found that the lower growth of E. coli K-12 (JM109) strain was the result of transcription inhibition of the osmoprotectant producing bet operon (betABT).

Conclusions: The transcriptional changes caused by the deletion of cra gene did not affect the activity of the central carbon metabolism, suggesting that Cra does not act alone; rather it interacts with other pleiotropic regulators to create a network of metabolic effects. An unexpected outcome of this work is the finding that cra deletion caused transcription inhibition of the bet operon in E. coli K-12 (JM109) but did not affect this operon transcription in E. coli B (BL21). This property, together with the insensitivity to high glucose concentrations, makes this the E. coli B (BL21) strain more resistant to environmental changes.
\end{abstract}

\section{Background}

Acetate accumulation is one of the main concerns during high cell density growth of $E$. coli [1,2]. It was established that acetate concentrations above $40 \mathrm{mM}(2.4 \mathrm{~g} / \mathrm{L})$ negatively affect cellular growth and recombinant protein production [3-5]. Acetate accumulation is dependent on the bacterial strain [6] and is affected by high growth rate and low oxygen concentration [4,7]. Methods have been developed to reduce acetate accumulation, including different glucose feeding strategies, usage of lower acetate

\footnotetext{
* Correspondence: yossi@nih.gov

+ Contributed equally

Full list of author information is available at the end of the article
}

producing carbon sources, and the development of mutant strains with altered acetic acid metabolic flux [8-10].

The acetic acid production pattern of $E$. coli $B$ (BL21) is different from that of E. coli K-12 (JM109) especially when the bacteria grow to high densities at high glucose concentrations [11]. E. coli K-12 (JM109) accumulates acetate up to $11 \mathrm{~g} / \mathrm{L}$ and its growth rate slows down; E. coli B (BL21) on the other hand, accumulates acetate to about $3 \mathrm{~g} / \mathrm{L}$ and its growth rate is not affected. Careful evaluation of these two strains revealed that E. coli $\mathrm{B}$ (BL21) has active glyoxylate shunt, gluconeogenesis, anaplerotic pathway, and TCA cycle compared with E. coli

\section{Biomed Central}


K-12 (JM 109) [12,13]. It seems that in E. coli B (BL21), the central carbon metabolism pathways associated with glucose consumption are operating at the same rate regardless of the glucose concentration. Based on the above finding, it was suggested that FruR is responsible for the difference in the glucose metabolism of these two E. coli strains.

FruR, also known as, Cra (Catabolic repressor/activator), is a global transcription regulatory protein in enteric bacteria that regulates gene expression by binding to a specific DNA sequence [14]. It was reported that the fruR gene modulates the direction of carbon flow in $E$. coli by transcriptional activation of genes that encode enzymes associated with oxidative and gluconeogenic carbon flow and by repression of genes that are associated with fermentative carbon flow $[15,16]$. Cra is a common activator of the gene set $p p s A, f b p, p c k A, a c e A$, which are vital for acetate uptake. Sugar catabolites tend to bind to $\mathrm{Cra}$ and displace it from the operator sites in target operons [16]. These catabolites are present at high concentrations during growth in the presence of sugars, but in low concentration during growth in the presence of gluconeogenic substances. Genes that are activated by Cra (e.g. ppsA, $p c k A$, and $a c e B A$ ), are generally subject to catabolite repression, while genes that are repressed by $\mathrm{Cra}$ (e.g. fru, pts, and $e d d$ ), are subjected to catabolite activation [16]. Most studies on the role of Cra in E.coli have used E.coli K-12 such as DH5 $\alpha$, BW25113, and MG1655 at low cell densities. The inactivation of Cra in plasmidbearing E. coli $\mathrm{DH} 5 \alpha$ altered metabolic gene expression, improved growth rate [17], and improved plasmid yield [18], while the deleting of $\mathrm{cra}$ gene had no distinct effect on the phenotype of E. coli BW25113 [19]. cra deletion in E. coli MG1655 contributed to accumulation of pyruvate by suppressing gluconeogenesis due to the decreased expression of $p p s A$ and $p c k A$ [20].

Considering what is currently known about Cra regulation, and the difference between $E$. coli K-12 (JM109) and E. coli B (BL21) in regard to glucose metabolism, it was suggested that in E. coli B (BL21) cra is constitutively expressed, which explains why acetate is not accumulated. In the present work, we have investigated the effect of $\mathrm{cra}$ deletion on the central carbon metabolism in E. coli B (BL21) and E. coli K-12 (JM109) to verify the above assumption and to understand further the function of Cra in high cell density at high glucose, by following growth kinetics and gene expression.

\section{Results}

Cells growth parameters of the cra-positive and the cranegative strains

Growth kinetics, glucose consumption and media conductivity profile of $E$. coli $\mathrm{B}$ (BL21) and E. coli K-12 (JM 109), without modification and with the $c r a$ gene deletion $\left(\mathrm{cra}^{-}\right)$, are shown in Figure 1 and 2, and acetate and pyurvate production kinetics are shown in Figure 3. cra deletion in E. coli B (BL21) minimally affected growth, final cell yield, glucose consumption and maximal acetate and pyruvate accumulation. Unlike E. coli B (BL21), cra deletion in E. coli K-12 (JM109) affected the cell growth pattern. The final $\mathrm{OD}_{600}$ was 30 in E. coli K-12 (JM109) and 18 in E. coli K-12 (JM109) $\mathrm{Cra}^{-}$, maximal acetate and pyruvate concentrations in E. coli K-12 (JM109) were $9 \mathrm{~g} / \mathrm{L}$ and $14 \mathrm{~g} / \mathrm{L}$ respectively, and in E. coli $\mathrm{K}-12$ (JM109) $\mathrm{cra}^{-}$acetate and pyruvate concentration reached $6.6 \mathrm{~g} / \mathrm{L}$ and $13 \mathrm{~g} / \mathrm{L}$ respectively.

Media conductivity values are shown in Figure 1 and 2 . The conductivity increased from $10 \mathrm{mS} / \mathrm{cm}$ to $11 \mathrm{mS} / \mathrm{cm}$ in E. coli B (BL21) and from $10 \mathrm{mS} / \mathrm{cm}$ to $13 \mathrm{mS} / \mathrm{cm}$ in E. coli B (BL21) $\mathrm{cra}^{-}$. In E. coli $\mathrm{K}-12$ (JM 109), the conductivity reached $20 \mathrm{mS} / \mathrm{cm}$ and in $E$. coli $\mathrm{K}-12$ (JM109) $\mathrm{cra}^{-}$, reached $21 \mathrm{mS} / \mathrm{cm}$.

\section{Effect of salt concentration on cell growth}

To evaluate the effect of the media conductivity (salt concentration) on the bacterial growth, E. coli B (BL21) with and without cra, and E. coli K-12 (JM 109) with and without $c r a$, were grown in regular LB media containing $5 \mathrm{~g} / \mathrm{L}$ (conductivity $10 \mathrm{mS} / \mathrm{cm}$ ) and in LB media containing $15 \mathrm{~g} / \mathrm{L} \mathrm{NaCl}$ (conductivity $22 \mathrm{mS} / \mathrm{cm}$ ).

The growth of the different strains and media conductivity are shown in Figure 4 and 5. Both E. coli B (BL21) strains were not affected by the high conductivity media both strain grew to an $\mathrm{OD}_{600}$ of 44 and the media conductivity reached $26-27 \mathrm{mS} / \mathrm{cm}$. However, there was a difference between the two E. coli K-12 strains; E.coli K-12 (JM109) grew to final $\mathrm{OD}_{600}$ of 30 in regular LB media and to $27 \mathrm{OD}_{600}$ in the high conductivity media, while $E$. coli $\mathrm{K}-12$ (JM109) $\mathrm{cra}^{-}$grew to final $\mathrm{OD}_{600}$ of 18 in the regular media and to $11 \mathrm{OD}_{600}$ in the high conductivity media. During the growth of both E. coli K-12 strains, the media conductivity reached $40 \mathrm{mS} / \mathrm{cm}$.

\section{Comparative transcription analysis by microarray and real time PCR}

Results of microarray analysis, expressed as $\log 2$ ratios of samples taken during the log phase growth from cra negative strains and parental ( $\mathrm{cra}$ - positive) stains, are shown in Figure 6 and 7. A positive ratio is an indication that gene transcription is higher in the cra -negative strains and negative ratio is an indication that the gene transcription is lower in the cra negative strain. The transcription comparison was done on the following pathways of the central carbon metabolism: the TCA cycle, the glyoxylate shunt, the gluconeogenesis, and the glycolysis. No significance transcription differences were identified in the glycolysis and the TCA cycle pathways (Data not shown) but differences were identified in the expressions 
(A)

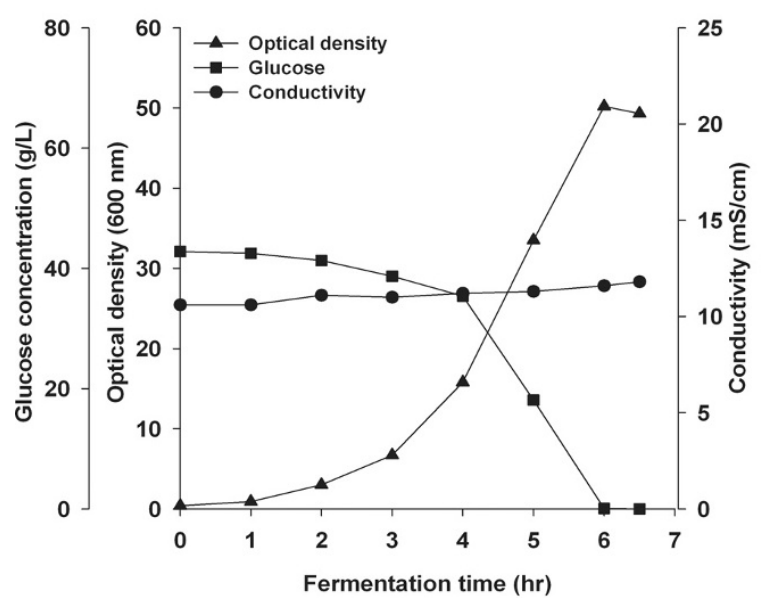

(B)

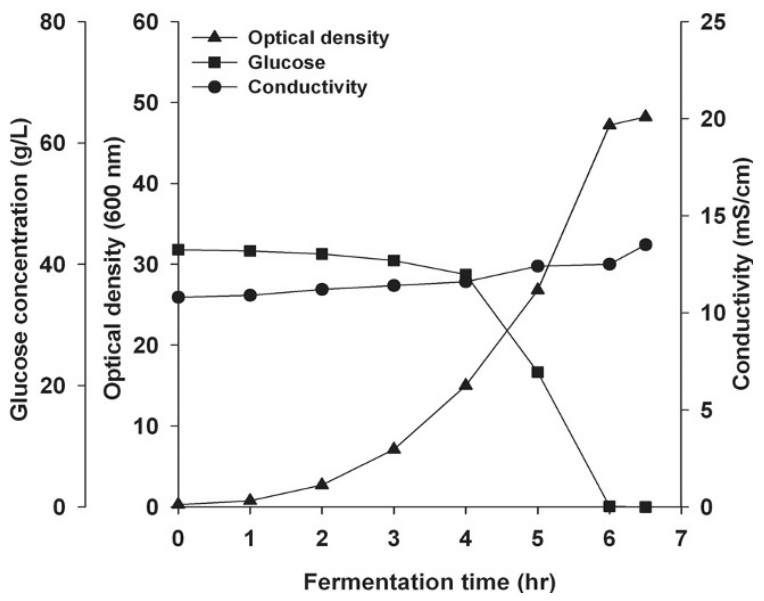

Figure 1 Growth, glucose consumption, and conductivity of E. coli B (BL21). (A) E. coli B (BL21), (B) E. coli B (BL21) cra-

of the glyoxylate shunt pathway genes (aceA and $a c e B$ ), the acetate producing gene $(p o x B)$, and the gluconeogenesis gene (ppsA); all these genes were down regulated in both $\mathrm{Cra}^{-}$strains while acetate uptake gene (acs) was down regulated significantly only in E.coli B (BL21) $\mathrm{cra}^{-}$.

The different response of the tested strains to high salt concentration (Figure 4 and 5) prompted us to look for transcription differences of the osmoprotectant bet operon genes (betABT): the results are shown in Figure 8. It is clear that the expression level of the bet operon genes (betABT) in E. coli $\mathrm{K}-12$ (JM109) $\mathrm{cra}^{-}$are low compared with their expression in the $\mathrm{cra}$ - positive stain. This information was confirmed by Real Time PCR (Additional file 1, Figure S2, A, B, and C).

\section{Discussion}

The purpose of this work was to find out if the global regulator $\mathrm{Cra}$ is responsible for the difference in glucose consumption and acetate production between $E$. coli K12 (JM109) and E. coli B (BL21) as a result of its effect on the central carbon metabolism [21]. Based on published data about different growth parameters and metabolic activities of the two strains [11], we concluded that Cra may be constitutively expressed in E. coli B (BL21)
(A)

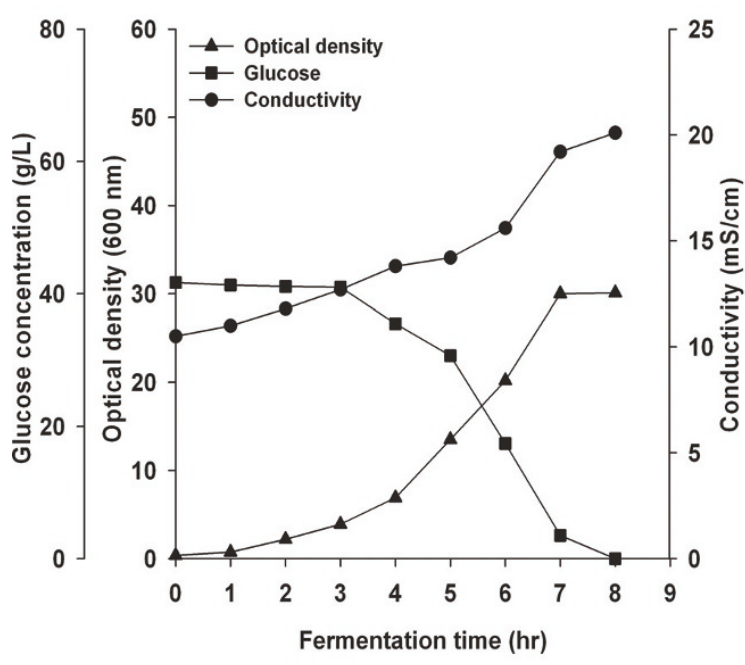

(B)



Figure 2 Growth, glucose consumption, and conductivity of E. coli K-12 (JM109). (A) E. coli K-12 (JM109), (B) E. coli K-12 (JM109) cra- . 


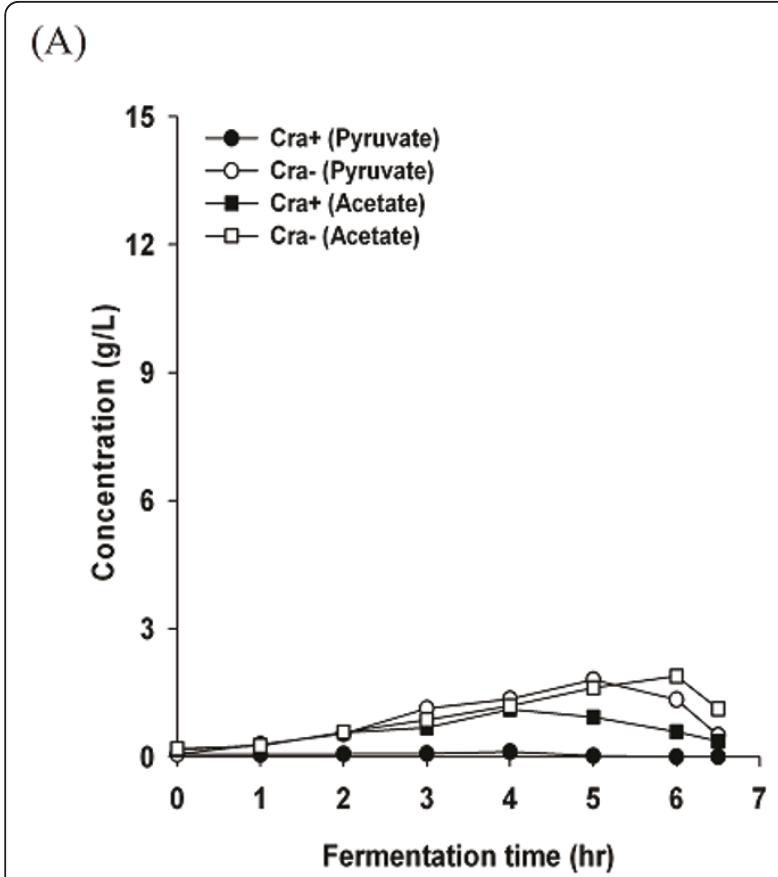

(B)

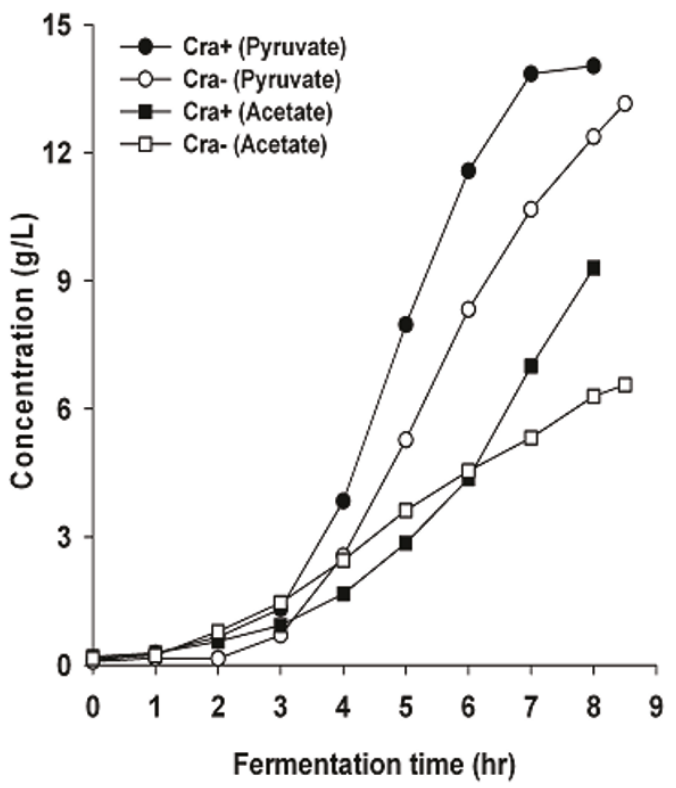

Figure 3 Pyruvate and acetate production of E. coli B (BL21) and E.coli K-12 (JM109). (A) E. coli B (BL21), (B) E. coli K-12 (JM109).

but not in E. coli K-12 (JM109) [7]. By creating cra deleted mutants of these two strains and by analyzing their growth, metabolism and gene expression, we anticipated to have more information on the role of the Cra regulating the central carbon metabolism in E. coli, and perhaps be able to create a strain tolerant to high glucose concentrations. The results showed that $\mathrm{cra}$ deletion in E.coli B (BL21) minimally affected the growth and the maximal acetate accumulation, while in E.coli K-12 (JM109), cra deletion showed considerable effect. E. coli K-12 (JM109) grew to an OD of 30 and accumulated $9.0 \mathrm{~g} / \mathrm{l}$ acetate, while E. coli K-12 (JM109) $\mathrm{Cra}^{-}$stopped growing as soon as acetate concentration reached $6.6 \mathrm{~g} / \mathrm{L}$ at an OD of 18 ; at this point, the media
(A)

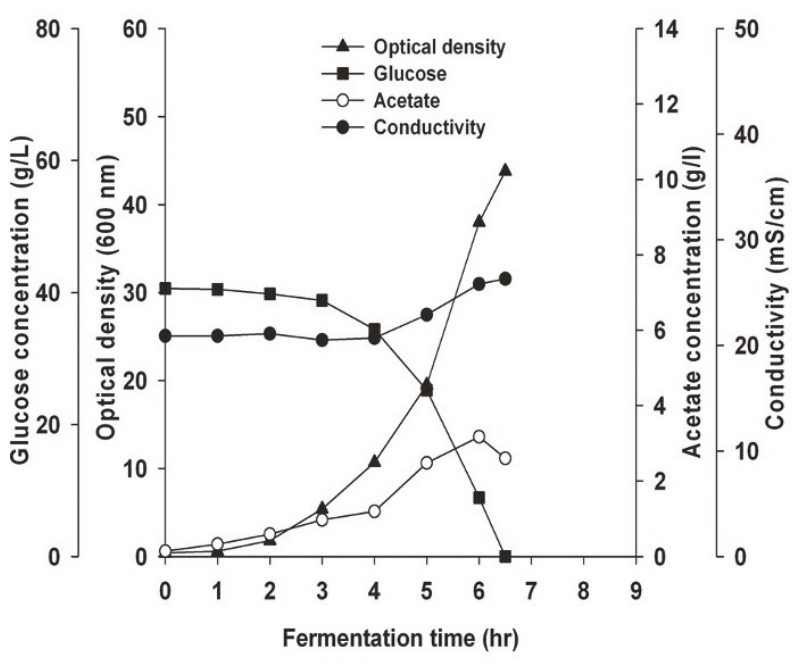

(B)

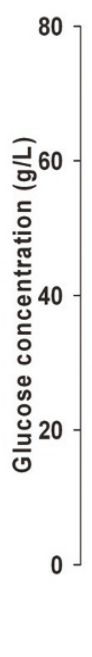

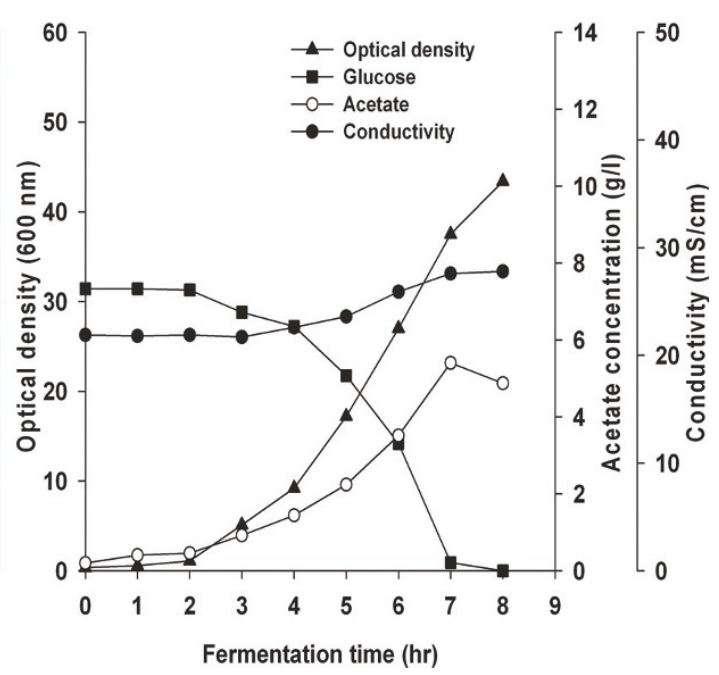

Figure 4 Growth, glucose consumption, conductivity, and acetate production of $E$. coli B (BL21) in LB media containing $15 \mathrm{~g} / \mathrm{L} \mathrm{NaCl}$. (A) E. coli B (BL21), (B) E. coli B (BL21) cra-. 
(A)

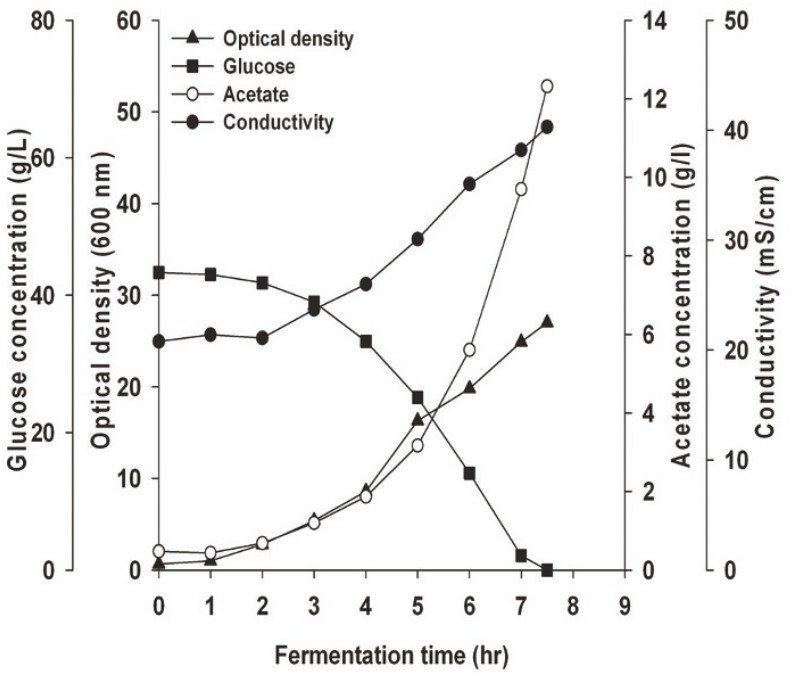

(B)

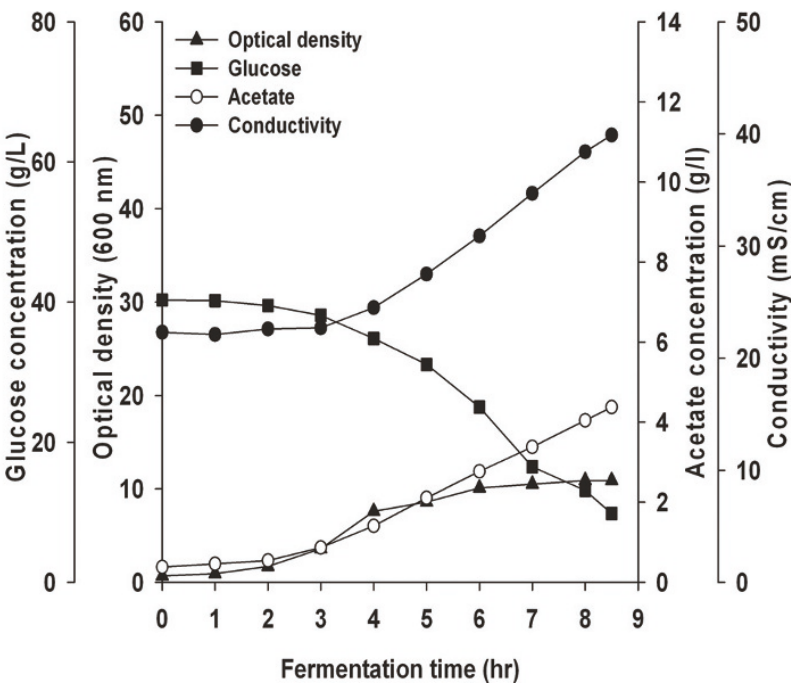

Fermentation time (hr)

Figure 5 Growth, glucose consumption, conductivity, and acetate production of E. coli K-12 (JM109) in LB media containing 15 g/L NaCl. (A) E. coli K-12 (JM109), (B) E. coli K-12 (JM109) $\mathrm{cra}^{-}$.

conductivity reached $21 \mathrm{mS} / \mathrm{cm}$, indicating a possible effect on the ability of the mutant cell to keep its osmolarity.

Our microarray results showed that cra deletion affected the transcription of genes associated with acetate metabolism. pps $A$ (gluconeogenesis gene), ace $A$, aceB (the glyoxylate shunt pathway genes) and $\operatorname{pox} B$ (the acetate producing gene) were all down-regulated in both knockout strains, while acs (acetate uptake gene) was down regulated only in E.coli B (BL21) $\mathrm{cra}^{-}$. These results are similar to previous reports that Cra represses transcription of genes that encode glycolytic (sugar-catabolizing) enzymes such as key enzymes in the Embden-Meyerhof and Enter-Doudoroff pathways, but activates transcription of genes that encode biosynthetic and oxidative enzymes such as key enzymes in the Krebs cycle, the glyoxylate shunt, the gluconeogenic pathway, and electron transfer [16]. Concerning the metabolic activity of the bacteria; the results presented here showed little or no effect of the cra deletion on acetate and pyruvate production. These

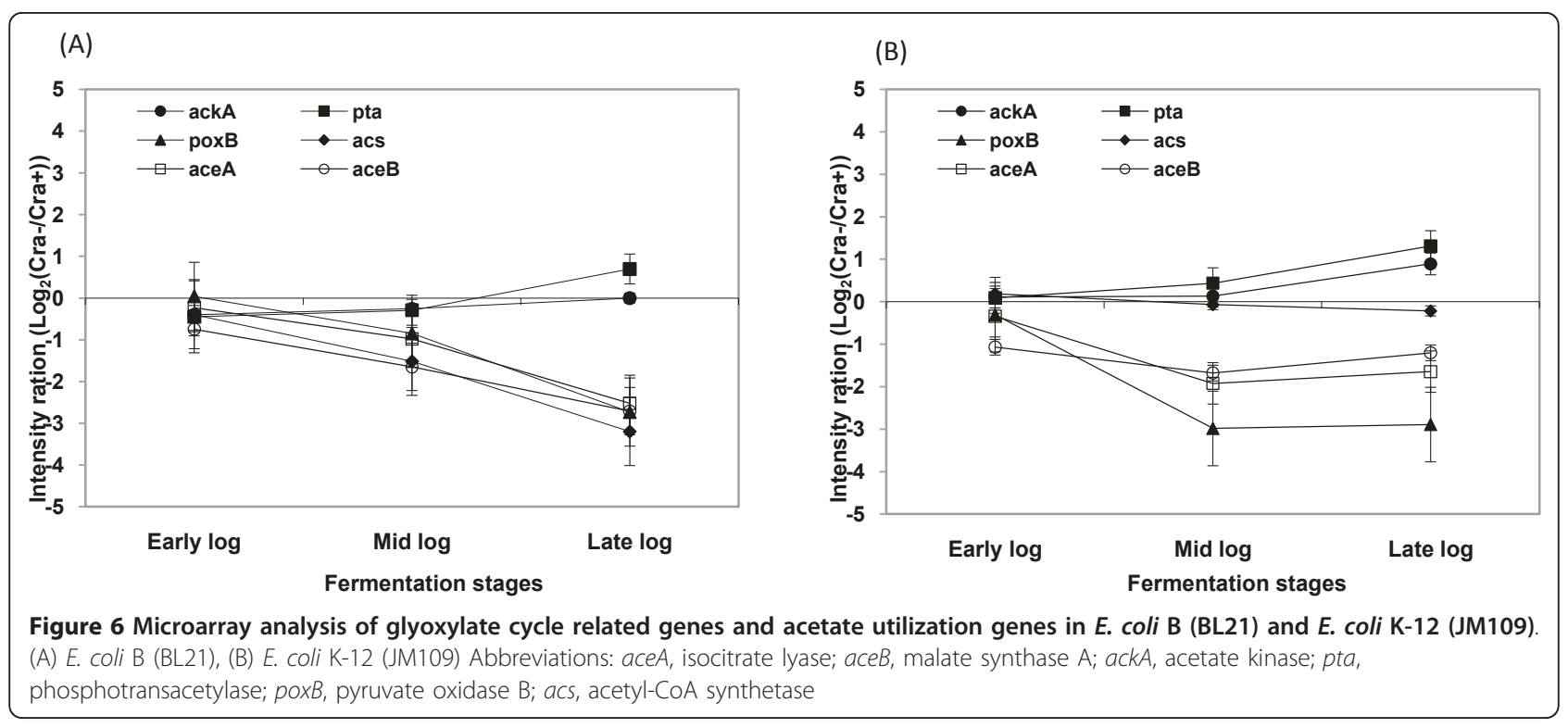




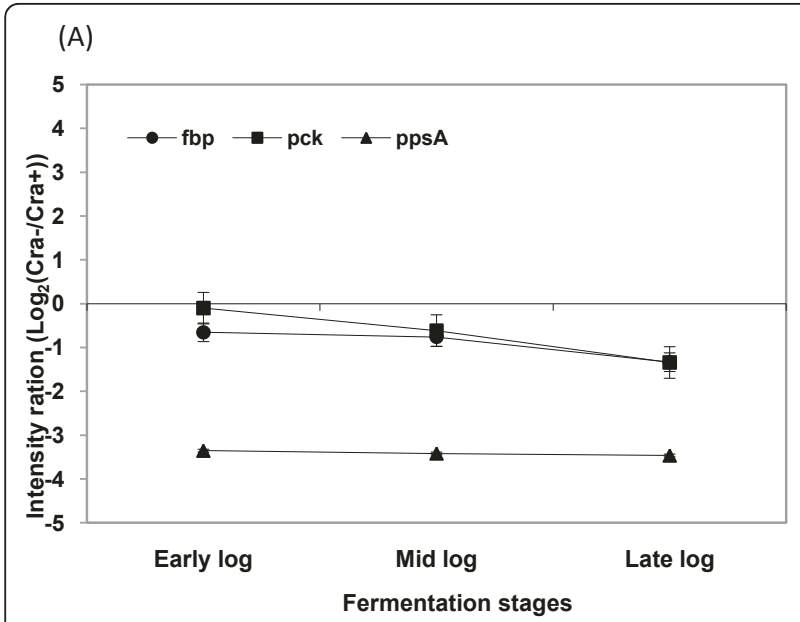

(B)

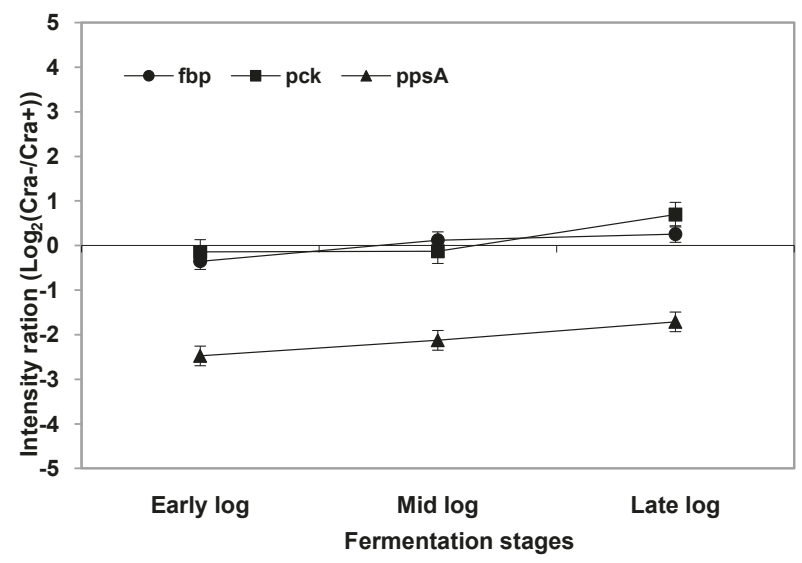

Figure 7 Microarray analysis of glucoenogensis related genes in E. coli B (BL21) and E. coli K-12 (JM109). (A) E. coli B (BL21), (B) E. coli K12 (JM109) Abbreviations: fbp, fructose-1,6-bisphosphatase; $p c k A$, phosphoenolpyruvate carboxykinase; ppsA, phosphoenolpyruvate synthase

observations are different from previous reports that Cra deletion in E. coli MG1655 contributed to accumulation of pyruvate by suppressing gluconeogenesis due to the down regulation of ppsA and pckA [20]. It is possible that Cra does not act alone, rather interacts with other pleiotropic regulators to create a network of metabolic effects [19].

Cra has been known to affect positively and negatively the transcription of several other genes such as $a c n A / B$ (aconitase), icdA (isocitrate dehydrogenase), eda (2-keto-3deoxy-6-phosphogluconate adolase), edd (6-phosphogluconate dehydrase), $p f k A$ (phosphofructokinase), and $p y k F$ (pyruvate kinase isoenzymes), but the transcription patterns of these genes in this work were not significantly different between the two strains (results not shown). Another global regulator that was reported to affect the glyoxylate shunt operon (aceBAK), is ArcA/B; this twocomponent system is considered to respond to oxygen limitation under microaerobic conditions [19]. In addition to $\mathrm{Cra}$ and $\mathrm{ArcA} / \mathrm{B}$, other metabolic regulators are involved in acetate metabolism in E.coli: acetyl-CoA synthetase is under the control of the sigma factors RpoS and RpoD and requires CAMP receptor protein, and the oxygen regulator (Fnr) for full expression [22]. Pyruvate oxydase B is also under the control of RpoS and cAMP-CRP. However, our work seems that $\mathrm{cra}$ deletion does not affect the transcription patterns of cyaA, crp and rpoD in E. coli B (BL21) and E. coli K-12 (JM109) (Additional file 1, Figure S1, A and B). As for the transcription of rpoS, the level decreased significantly only at the late log phase in E. coli B (BL21) $\mathrm{cra}^{-}$ but not in E. coli K-12 (JM109) $\mathrm{cra}^{-}$. The down regulation of rpoS was reported to result in the high transcription of poxB and the low transcription of acs in E. coli B (BL21) [7].

An unexpected outcome of our work is the discovery that the bet operon, which has a role in protecting E. coli against high osmotic pressure [23-26], was down-regulated in E. coli K -12 (JM109) $\mathrm{cra}^{-}$strain. This phenomenon can provide a possible explanation to the growth inhibition of this strain when the media conductivity was above $18 \mathrm{mS} / \mathrm{cm}$. The bet operon consists of three genes: bet $T$ (choline transporter), betA (choline dehydrogenase), and betB (betaine aldehyde dehydrogenase) [27]. The Choline transporter has high affinity for choline and transports choline into cell; choline dehydrogenase is an oxygen-dependent enzyme that converts choline into betaine aldehyde; and betaine aldehyde dehydrogenase converts betaine aldehyde into glycinebetaine [28]. The bet genes are induced by choline, oxygen, and osmotic stress [29]. The inhibition of cell growth that was observed only in E. coli $\mathrm{K}-12$ (JM109) $\mathrm{cra}^{-}$is likely the result of the limited transcription of the bet operon (Figure 8). The cra deletion affected the bet operon expression only in E. coli $\mathrm{K}-12$ (JM109) and not in E. coli B (BL21); it is possible that Cra affects the bet operon indirectly. This is an additional difference between these two strains that may point to less regulation in E. coli B. The insensitivity of $E$. coli B (BL21) to the cra deletion, together with its insensitivity to high glucose concentration, enables this strain to be less affected by environmental stress such as glucose concentration and ionic strength. This may be the results of lacking an internal control mechanism that may be responsible for E. coli K12 inability to cope with environmental stress.

\section{Conclusions}

To evaluate the role of the global regulator Cra in the central carbon metabolism of E. coli $\mathrm{K}$ and $E$. coli $\mathrm{B}$, the cra gene was deleted and the mutant strains were compared to the parental strains in their gene expression, growth and metabolic behaviour. The transcriptional 

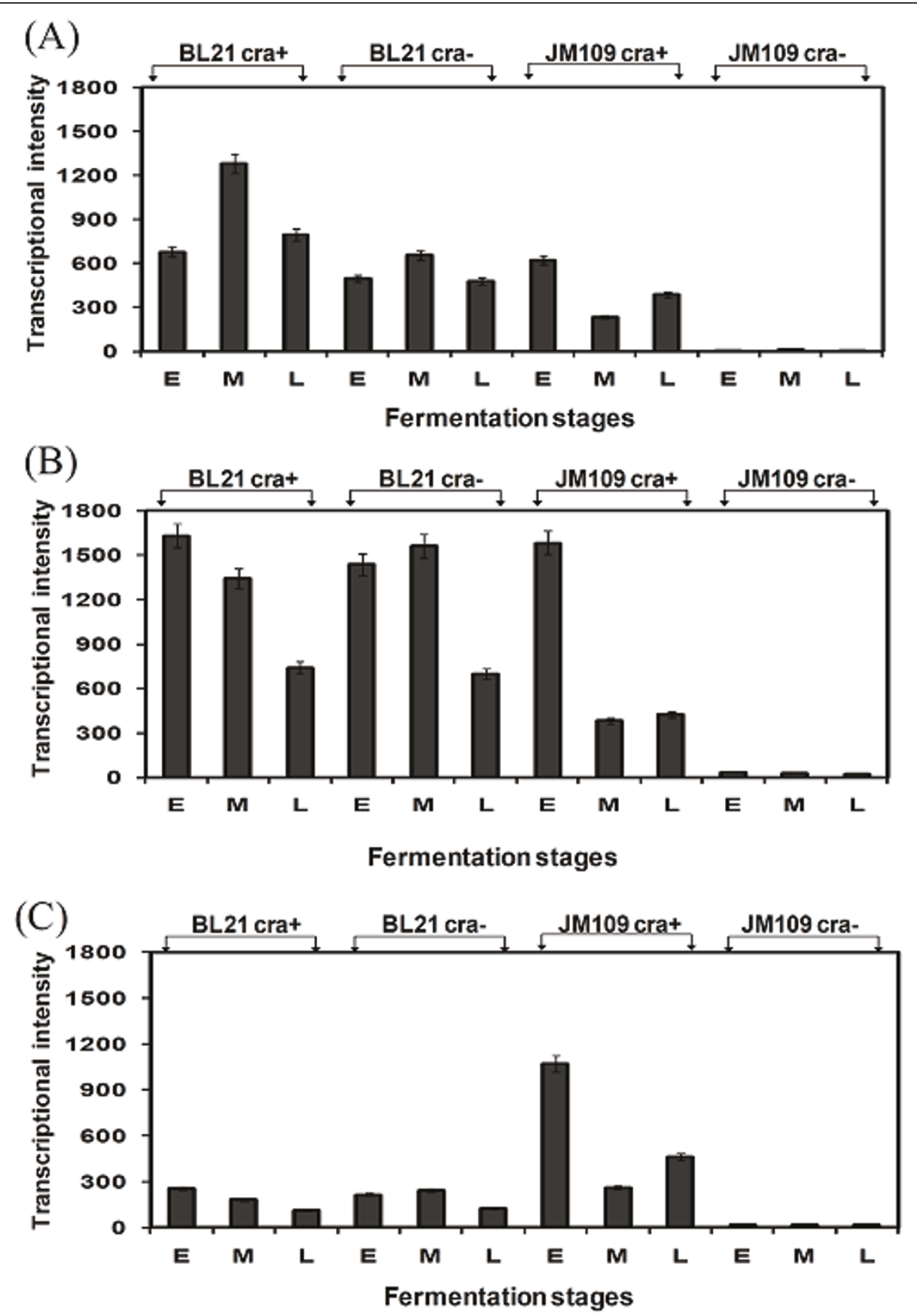

Figure 8 Microarray analysis of bet operon genes in E. coli B (BL21) and E. coli K-12 (JM109). (A) betT, choline transporter, (B) betA, choline dehydrogenase, and $(C)$ bet $B$, betain aldehyde dehydrogenase.

changes caused by the deletion of cra gene support the assumption that Cra is a major regulatory molecule that has an effect on the expression of $p p s A$, aceBAK and acs in E.coli B (BL21), but these transcriptional changes did not affect the activity of the central carbon metabolism, suggesting that $\mathrm{Cra}$ does not act alone, rather it interacts with other pleiotropic regulators to create a network of metabolic effects. An unexpected outcome of this work is the finding that cra deletion in E. coli K-12 (JM109) caused transcription inhibition of the bet operon which is responsible for maintaining the salt concentration in the cells. In comparison, the bet operon expression was not affected in E. coli B (BL21). This property, together with this strain insensitivity to high glucose concentrations, 
makes this strain more resistant to environmental changes and may be one of the reasons for the growth properties of this strain.

\section{Materials and methods \\ Bacterial strains}

E. coli B (BL21) ( $\lambda \mathrm{DE} 3)\left(\mathrm{F}^{-}\right.$, omp T, hsdSB (rB-, $\left.m B+\right)$, dcm, gal, Cmr) and E. coli K-12 (JM109) ( $\lambda \mathrm{DE} 3$ ) (endA1, recA1, gyrA96, thi, hsdR17 (rk-, mk+), relA1, supE44, $\Delta \lambda-, \Delta$ (lacpro $A B)$, F', traD36, pro $A B$, lacIqZ $\triangle M 15$ ) were used. Both strains were obtained from Promega Corp. (Madison, WI).

cra gene knock-out was carried out by recombination using plasmid pKD46 as previously described [30]. A kanamycin marker in plasmid pACYC177 (New England Biolab, Inc., Beverly, MA) was amplified by PCR to disrupt cra gene using two primers 5'-GTGAAACTGGATGA AATCGCTCGGCTGGCGGGAGTGTCGCGGACCACT GCTACGGTCTGCGTTGTCGGGAAGATGCG and 5'CTTAGCTACGGCTGAGCACGCCGCGGCGATAGAGATTACGTTTAATGCGCGTACAAAGCCGCCGTC CCGTCAAGTCA. PCR products carrying kanamycin marker and homologous region (50 bp) were electroporated into JM109 (DE3) harboring pKD46 where lambda recombinase was fully expressed by L-arabinose during culture at $30^{\circ} \mathrm{C}$, and subsequently cells were spread on LB agar containing kanamycin. Candidate colonies were grown at $42^{\circ} \mathrm{C}$ to cure plasmid pKD46 carrying the temperature sensitive origin of replication. Specific gene knock-out in JM109(DE3) $\mathrm{cra}^{-}$strain was confirmed by PCR with primers which can hybridize upstream and downstream of deleted cra genes. The PCR products were also electroporated into MG1655 harboring pKD46 to make MG1655 $\mathrm{cra}^{-}$strain. P1 lysates of MG1655 $\mathrm{cra}^{-}$were used to transduce cra mutation carrying a kanamycin marker into BL21(DE3) strain to make BL21(DE3) $\mathrm{cra}^{-}$which were confirmed as described above.

\section{Fermentation and sample preparation}

The four strains were grown at $37^{\circ} \mathrm{C}$ in modified LB medium containing $10 \mathrm{~g} / \mathrm{L}$ tryptone, $5 \mathrm{~g} / \mathrm{L}$ yeast extract $(15 \mathrm{~g} /$ $\mathrm{L}$ for JM109), $5 \mathrm{~g} / \mathrm{L} \mathrm{NaCl}$, and $5 \mathrm{~g} / \mathrm{L} \mathrm{K}_{2} \mathrm{HPO}_{4}$. After sterilization, $10 \mathrm{mM} \mathrm{MgSO}$, $1 \mathrm{ml} / \mathrm{L}$ trace metal solution [31], and $40 \mathrm{~g} / \mathrm{L}$ glucose were added. Overnight cultures grown at $37^{\circ} \mathrm{C}$ were used to inoculate $3.0 \mathrm{~L}$ of medium in a $\mathrm{B}$. Braun fermentor equipped with data acquisition and a control system. The cultures were grown to high-cell-density, the $\mathrm{pH}$ was controlled at 7.0 by the addition of $50 \%$ $\mathrm{NH}_{4} \mathrm{OH}$, and dissolved oxygen was kept at $30 \%$ air saturation. For high-conductivity media, the $\mathrm{NaCl}$ was adjusted to $15 \mathrm{~g} / \mathrm{L}$, the other compositions were the same. Samples for acetic acid, pyruvate, glucose concentration, and conductivity analysis were collected at regular intervals, centrifuged at $14,000 \times \mathrm{g}$ for $5 \mathrm{~min}$ and the supernatant was analyzed with HPLC. Samples for total RNA purification were collected and centrifuged at 14,000 $\times \mathrm{g}$ for $1 \mathrm{~min}$ at $4^{\circ} \mathrm{C}$, the supernatant was removed, and the cell pellets were quickly frozen in dry ice and stored at $-80^{\circ} \mathrm{C}$.

\section{Analytical methods}

The concentrations of acetate and pyruvate were analyzed at $35^{\circ} \mathrm{C}$ (for acetate) and $65^{\circ} \mathrm{C}$ (for pyruvate) on an HP 1100 series (Agilent, Santa Clara, CA) using an Aminex HPX-87H column $(300 \times 7.8 \mathrm{~mm}$; Bio-Rad, CA, USA). The mobile phase was $0.008 \mathrm{~N}$ sulfuric acid and flow rate was $0.6 \mathrm{~mL} / \mathrm{min}$. After injection of a $20-\mu \mathrm{L}$ sample, absorbance was monitored at a wavelength of $210 \mathrm{~nm}$ using a UV detector. Glucose in the culture supernatant was determined using an YSI glucose analyzer (YSI Inc., Yellow Springs, OH). Conductivity was analyzed at $30^{\circ} \mathrm{C}$ using CDM210 conductivity meter (Radiometer Analytical, Lyon, France).

\section{Total RNA preparation and microarrays}

Total RNA was isolated using a MasterPure RNA Purification Kit (Epicentre Technologies, Madison, WI) and RNeasy Mini Kit (Qiagen, Valencia, CA) according to the manufacturers' protocols (MCR 85102 \& 74104). RNA concentration and purity were determined by measuring absorbance at $260 \mathrm{~nm}\left(\mathrm{~A}_{260}\right)$ using a NanoDrop 1000 (Thermo Scientific, Waltham, MA). Three biological replicates of RNA samples from E. coli B (BL21) and E. coli K-12 (JM109) cra knockout strain and wildtype were hybridized to Affymetrix E. coli Genome 2.0 Array. DNA-chip Analyzer (dChip version 2006) was used to process the raw data generated at the NIDDK Genomics Laboratory.

\section{Real-time PCR}

Reverse transcription (RT) was carried out with the High Capacity RNA-to-cDNA Kit using the manufacturer's protocol (Applied Biosystems). A 20-ul RT reaction mixture included $2 \mathrm{ug}$ of total RNA, $10 \mathrm{ul}$ of $2 \mathrm{X}$ RT buffer, 1 ul of 20X RT enzyme mix, and DEPC-treated water. Real-time PCRs were carried out on a 7900 HT Fast Real-Time PCR System (Applied Biosystems) with the Power SYBR Green PCR Master Mix (Applied Biosystems). The PCR mixtures were incubated at $95^{\circ} \mathrm{C}$ for 10 min to activate the AmpliTaq DNA polymerase, followed by 40 cycles of amplification $\left(95^{\circ} \mathrm{C}\right.$ for $15 \mathrm{sec} ; 60^{\circ} \mathrm{C}$ for $1 \mathrm{~min}$ ). A final extension step was performed at $60^{\circ} \mathrm{C}$ for $10 \mathrm{~min}$. Real-time PCR results were also analyzed using the 7900 HT SDS software (Applied Biosystems).

\section{Additional material}

Additional file 1: Supplemental Figure S1 and S2. Microarray data (Supplemental Figure S1) and real time PCR data (Supplemental Figure S2). 


\section{Acknowledgements}

Funding was provided by the intramural program of the National Institute of Diabetes and Digestive and Kidney Diseases, National Institutes of Health. The authors would like to thank Mrs. D. Livnat for critical review of the manuscript.

\section{Author details}

'Biotechnology Core Laboratory, NIDDK, NIH, Bldg 14A Room 173, Bethesda, Maryland, 20892, USA. 'Industrial Biotechnology and Bioenergy Research Center, KRIBB, Daejeon, 305-806, Korea.

\section{Authors' contributions}

YS performed experiments and analysis, literature search and writing the manuscript, JP hypothesised the idea, survey the literature, analyze results and writing the manuscript, LT performed fermentation experiments, SL genetic work, JS conceived the study participated in the designed and coordination and the writing of the manuscript. All authors read and approved the final manuscript.

\section{Competing interests}

The authors declare that they have no competing interests.

Received: 20 January 2011 Accepted: 30 June 2011

Published: 30 June 2011

\section{References}

1. Eiteman MA, Altman E: Overcoming acetate in Escherichia coli recombinant protein fermentations. Trends Biotechnol 2006, 24:530-536.

2. Shiloach J, Rinas U: Glucose and acetate metabolism in E. coli - System level analysis and biotechnological applications in protein production processes. In Systems biology and biotechnology of Escherichia coli. Edited by: Lee SY. Springer Science; 2009:377-400.

3. Kleman GL, Strohl WR: Acetate metabolism by Escherichia coli in highcell-density fermentation. Appl Environ Microbiol 1994, 60:3952-3958.

4. Ko YF, Bentley WE, Weigand WA: The effect of cellular energetics on foreign protein production. Appl Biochem Biotechnol 1995, 50:145-159.

5. Kim JY, Cha HJ: Down-regulation of acetate pathway through antisense strategy in Escherichia coli: improved foreign protein production. Biotechnol Bioeng 2003, 83:841-853.

6. van de Walle M, Shiloach J: Proposed mechanism of acetate accumulation in two recombinant Escherichia coli strains during high density fermentation. Biotechnol Bioeng 1998, 57:71-78.

7. Phue JN, Shiloach J: Impact of dissolved oxygen concentration on acetate accumulation and physiology of $E$. coli BL21, evaluating transcription levels of key genes at different dissolved oxygen conditions. Metab Eng 2005, 7:353-363.

8. De Mey M, De Maeseneire S, Soetaert W, Vandamme E: Minimizing acetate formation in E. coli fermentations. J Ind Microbiol Biotechnol 2007, 34:689-700.

9. Lee SY: High cell-density culture of Escherichia coli. Trends Biotechnol 1996, 14:98-105

10. Castaño-Cerezo S, Pastor JM, Renilla S, Bernal V, Iborra JL, Cánovas M: An insight into the role of phosphotransacetylase (pta) and the acetate/ acetyl-CoA node in Escherichia coli. Microb Cell Fact 2009, 8:54.

11. Phue JN, Shiloach J: Transcription levels of key metabolic genes are the cause for different glucose utilization pathways in E. coli B (BL21) and E. coli K (JM109). J Biotechnol 2004, 109:21-30.

12. Noronha SB, Yeh HJ, Spande TF, Shiloach J: Investigation of the TCA cycle and the glyoxylate shunt in Escherichia coli BL21 and JM109 using (13)CNMR/MS. Biotechnol Bioeng 2000, 68:316-327.

13. Phue JN, Noronha SB, Hattacharyya R, Wolfe AJ, Shiloach J: Glucose metabolism at high density growth of E. coli B and E. coli K: differences in metabolic pathways are responsible for efficient glucose utilization in E. coli B as determined by microarrays and Northern blot analyses. Biotechnol Bioeng 2005, 90:805-820.

14. Ramseier TM, Negre D, Cortay JC, Scarabel M, Cozzone AJ, Saier MH Jr: In vitro binding of the pleiotropic transcriptional regulatory protein, FruR, to the fru, pps, ace, pts and icd operons of Escherichia coli and Salmonella typhimurium. J Mol Biol 1993, 234:28-44.
15. Ramseier TM, Bledig S, Michotey V, Feghali R, Saier MH Jr: The global regulatory protein FruR modulates the direction of carbon flow in Escherichia coli. Mol Microbiol 1995, 16:1157-1169.

16. Saier MH Jr, Ramseier TM: The catabolite repressor/activator (Cra) protein of enteric bacteria. J Bacteriol 1996, 178:3411-3417.

17. Ow DS, Lee RM, Nissom PM, Philp R, Oh SK, Yap MG: Inactivating FruR global regulator in plasmid-bearing Escherichia coli alters metabolic gene expression and improves growth rate. J Biotechnol 2007. 131:261-269.

18. Ow DS, Yap MG, Oh SK: Enhancement of plasmid DNA yields during fedbatch culture of a fruR-knockout Escherichia coli strain. Biotechnol Appl Biochem 2009, 52:53-59.

19. Perrenoud A, Sauer U: Impact of global transcriptional regulation by ArcA, ArcB, Cra, Crp, Cya, Fnr, and Mlc on glucose catabolism in Escherichia coli. J Bacteriol 2005, 187:3171-3179.

20. Sakai T, Nakamura N, Umitsuki G, Nagai K, Wachi M: Increased production of pyruvic acid by Escherichia coli RNase G mutants in combination with cra mutations. Appl Microbiol Biotechnol 2007, 76:183-192.

21. Sarkar D, Siddiquee KA, Arauzo-Bravo MJ, Oba T, Shimizu K: Effect of cra gene knockout together with edd and iclR genes knockout on the metabolism in Escherichia coli. Arch Microbiol 2008, 190:559-571.

22. Kumari S, Simel EJ, Wolfe AJ: $\sigma 70$ is the principal sigma factor responsible for transcription of acs, which encodes acetyl Coenzyme A synthetase in Escherichia coli. J Bacteriol 2000, 182:551-554.

23. Le Rudulier D, Strom AR, Dandekar AM, Smith LT, Valentine RC: Molecular biology of osmoregulation. Science 1984, 224:1064-1068.

24. Landfald B, Strom AR: Choline-glycine betaine pathway confers a high level of osmotic tolerance in Escherichia coli. J Bacteriol 1986, 165:849-855.

25. Styrvold OB, Falkenberg P, Landfald B, Eshoo MW, Bjornsen T, Strom AR: Selection, mapping, and characterization of osmoregulatory mutants of Escherichia coli blocked in the choline-glycine betaine pathway. J Bacteriol 1986, 165:856-863.

26. Perroud B, Le Rudulier D: Glycine betaine transport in Escherichia coli: osmotic modulation. J Bacteriol 1985, 161:393-401.

27. Osteras M, Boncompagni E, Vincent N, Poggi MC, Le Rudulier D: Presence of a gene encoding choline sulfatase in Sinorhizobium meliloti bet operon: choline-O-sulfate is metabolized into glycine betaine. Proc Natl Acad Sci USA 1998, 95:11394-11399.

28. Andresen PA, Kaasen I, Styrvold OB, Boulnois G, Strom AR: Molecular cloning, physical mapping and expression of the bet genes governing the osmoregulatory choline-glycine betaine pathway of Escherichia coli. J Gen Microbiol 1988, 134:1737-1746.

29. Lamark T, Rokenes TP, McDougall J, Strom AR: The complex bet promoters of Escherichia coli: regulation by oxygen (ArCA), choline (Betl), and osmotic stress. J Bacteriol 1996, 178:1655-1662.

30. Datsenko KA, Wanner BL: One-step inactivation of chromosomal genes in Escherichia coli K-12 using PCR products. Proc Natl Acad Sci USA 2000, 97:6640-6645.

31. Bauer S, Shiloach J: Maximal exponential growth rate and yield of E. coli obtainable in a bench-scale fermentor. Biotechnol Bioeng 1974, 16:933-941.

doi:10.1186/1475-2859-10-52

Cite this article as: Son et al:: The role of $\mathrm{Cra}$ in regulating acetate excretion and osmotic tolerance in E. coli K-12 and E. coli B at high density growth. Microbial Cell Factories 2011 10:52. 